\title{
Atomistic modelling as a complementary tool for diffraction studies
}

\author{
Max Avdeev \\ Australian Nuclear Science and Technology Organisation, Lucas Heights, Australia; \\ max@ansto.gov.au
}

Diffraction experiments typically provide clear picture of a crystal structure and basis for understanding material properties. However, for materials with high static or dynamic disorder and/or weakly occupied atomic sites, e.g. ionic conductors, the diffraction data reflecting space- and time-averaged state may struggle to distinguish several alternative models yielding similar $\chi^{2}$. In that case, atomistic modelling may help not only to identify the more energetically stable configuration but also provide insights into the mechanism of its formation. I will present several recent examples of studies of disordered oxide-ion and proton conductors, where $a b$ initio static and geometry optimisation calculations and molecular dynamics simulations not only helped to validate neutron diffraction analysis but also revealed the mechanism driving the disorder.

Keywords: Diffraction, crystal structure, atomistic modelling, ionic conductors. 